\title{
Benchmarking the (1+1) Evolution Strategy with One-Fifth Success Rule on the BBOB-2009 Function Testbed
}

\author{
Anne Auger \\ TAO Team, INRIA Saclay lle-de-France \\ LRI, Bat 490 Univ. Paris-Sud \\ 91405 Orsay Cedex France \\ Anne.Auger@inria.fr
}

\begin{abstract}
In this paper, we benchmark the $(1+1)$ Evolution Strategy (ES) with one-fifth success rule which is one of the first and simplest adaptive search algorithms proposed for optimization. The benchmarking is conducted on the noise-free BBOB-2009 testbed. We implement a restart version of the algorithm and conduct for each run $10^{6}$ times the dimension of the search space function evaluations.
\end{abstract}

\section{Categories and Subject Descriptors}

G.1.6 [Numerical Analysis]: Optimization-global optimization, unconstrained optimization; F.2.1 [Analysis of Algorithms and Problem Complexity]: Numerical Algorithms and Problems

\section{General Terms}

Algorithms

\section{Keywords}

Benchmarking, Black-box optimization, Evolutionary computation, Evolution Strategies, One-Fifth success rule, adaptive search

\section{INTRODUCTION}

Soon after the introduction of the pure random search as a stochastic optimization algorithm [2], it was recognized that adaptive algorithms where the sampling distribution is adapted (as opposed to pure random search) during the course of the optimization are necessary for efficient optimization. One of the oldest adaptive search algorithms adapts the step-length (or step-size) using the following idea: the step-size is increased after a successful step and decreased after a failure so as to maintain a success probability of approximately $1 / 5$, increase the step-size if the success probability is larger than $1 / 5$ and decrease it otherwise. The

Permission to make digital or hard copies of all or part of this work for personal or classroom use is granted without fee provided that copies are not made or distributed for profit or commercial advantage and that copies bear this notice and the full citation on the first page. To copy otherwise, to republish, to post on servers or to redistribute to lists, requires prior specific permission and/or a fee.

GECCO'09, July 8-12, 2009, Montréal Québec, Canada.

Copyright 2009 ACM 978-1-60558-505-5/09/07 ...\$5.00. discovery of this idea, known in the field of evolutionary algorithms as the one-fifth success rule, dates back to 1968 , introduced by Schumer and Steiglitz in [10] and discovered independently by others $[9,3]$.

In this paper, the $(1+1)$ Evolution Strategy (ES) with onefifth success rule and restart mechanism is benchmarked on the BBOB-2009 function testbed.

\section{THE (1+1)-ES WITH INDEPENDENT RESTARTS}

In this section we describe the $(1+1)$-ES with one-fifth success rule and independent restarts. We start by describing the $(1+1)$-ES with one-fifth success rule.

\subsection{The (1+1)-ES with $1 / 5$ success rule}

The (1+1)-ES with one-fifth success rule implements the idea that the step-size should increase if "too many" steps are successful, indicating that the search is too local and should decrease if "too few" steps are successful, indicating that the step-length used for sampling solutions is "too large". Optimally, the probability to sample successful steps should be close to one-fifth $[10,9]$.

The algorithm tested here and presented in Table 1 is the version of the one-fifth success rule presented in [8]. We consider a scalar objective function $f: \mathbb{R}^{D} \mapsto \mathbb{R}, \boldsymbol{x} \mapsto f(\boldsymbol{x})$ to be minimized and denote $\boldsymbol{X}_{n} \in \mathbb{R}^{D}$ the estimate of the solution (also called parent) at iteration $n$, and $\sigma_{n} \in \mathbb{R}_{+}$the step-size. A new solution (or offspring) $\widetilde{\boldsymbol{X}}_{n}$ is sampled by adding to $\boldsymbol{X}_{n}$ a spherical multivariate normal distribution centered at zero and scaled by the step-size $\sigma_{n}$ (Table 1 , line 3), i.e.,

$$
\widetilde{\boldsymbol{X}}_{n}=\boldsymbol{X}_{n}+\sigma_{n} \mathcal{N}(\mathbf{0}, \boldsymbol{I}),
$$

where $\mathcal{N}(\mathbf{0}, \boldsymbol{I})$ denotes a multivariate normal distribution with mean vector $\mathbf{0}$ and identity covariance matrix ${ }^{1}$. The objective function value of $\widetilde{\boldsymbol{X}}_{n}$ is computed and $\boldsymbol{X}_{n+1}$ equals $\widetilde{\boldsymbol{X}}_{n}$ if $f\left(\widetilde{\boldsymbol{X}}_{n}\right) \leq f\left(\boldsymbol{X}_{n}\right)^{2}$ or $\boldsymbol{X}_{n}$ otherwise, i.e., the best among offspring and parent is becoming the parent for the next iteration ${ }^{3}$.

\footnotetext{
${ }^{1}$ The associated density is such that lines with equal density are hyperspheres.

${ }^{2}$ We assume minimization.

${ }^{3}$ This explains the notation $(1+1)$ referring to the fact that at each iteration, there is a single parent (the first "1"), a single offspring (the second "1") and the best, i.e., the one having the smallest objective value, among the parent plus the offspring is kept for the next iteration.
} 
Table 1: Pseudo-code for the $(1+1)-E S$ with onefifth success rule.

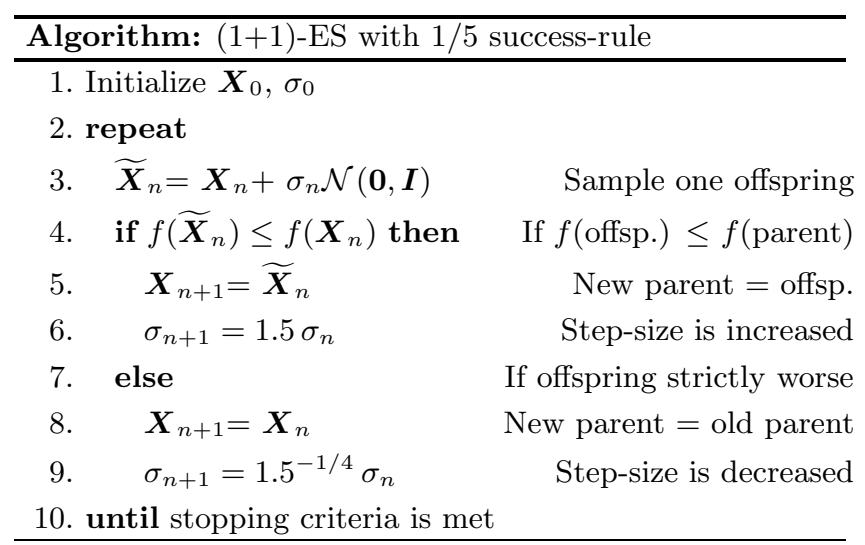

The step-size is increased by a factor 1.5 if the offspring is successful, i.e., if its objective function value is smaller or equal than the one of its parent (Table 1, line 6) and is decreased by a factor $1.5^{-1 / 4}$ otherwise (Table 1 , line 9 ). We now sketch why such a choice for the increasing and decreasing factors implements the idea of the one-fifth success rule. If the probability to sample a successful offspring from a given parent with step-size $\sigma_{n}$ is $1 / 5$, then the probability to sample an unsuccessful offspring equals $4 / 5$ and on average

$$
E\left(\sigma_{n+1} \mid \sigma_{n}\right)=\left((1.5)^{-1 / 4}\right)^{4 / 5}(1.5)^{1 / 5} \sigma_{n} .
$$

Since the term $\left((1.5)^{-1 / 4}\right)^{4 / 5}(1.5)^{1 / 5}$ equals 1 we hence have

$$
E\left(\sigma_{n+1} \mid \sigma_{n}\right)=\sigma_{n} .
$$

Furthermore, if the probability of sampling successful offspring, that we denote $p_{s}$ is larger than $1 / 5$, on average the step-size will satisfy

$$
E\left(\sigma_{n+1} \mid \sigma_{n}\right)=\left((1.5)^{-1 / 4}\right)^{1-p_{s}}(1.5)^{p_{s}} \sigma_{n}
$$

and thus the step-size will increase on average since the term

$$
\left((1.5)^{-1 / 4}\right)^{1-p_{s}}(1.5)^{p_{s}}
$$

is strictly larger 1 . The same reasoning holds if $p_{s}$ is smaller than $1 / 5$ implying that the step-size will decrease on average.

We have tested two other variants that are given in Table 2 and Table 3 . In Variant 1, in case of equality between the objective function values of the offspring and parent the step-size stays constant and the current estimate of the solution $\boldsymbol{X}_{n}$ stays constant as well. In the second variant (Table 3), in case of equality between the objective function values of the offspring and parent, the step-size stays constant but the $\boldsymbol{X}_{n+1}$ takes the value of the offspring $\widetilde{\boldsymbol{X}}_{n}$.

\subsection{The independent-restart (1+1)-ES}

We have implemented an independent-restart version of the $(1+1)$-ES: for each start the initial solution $\boldsymbol{X}_{0}$ is sampled uniformly in $[-4,4]^{D}$ and the step-size $\sigma_{0}$ is initialized at 2. After reaching a stopping criteria (described in the next section) the algorithm is (re-)initialized and restarted.
Table 2: Pseudo-code for the $(1+1)-E S$ with onefifth success rule Variant 1.

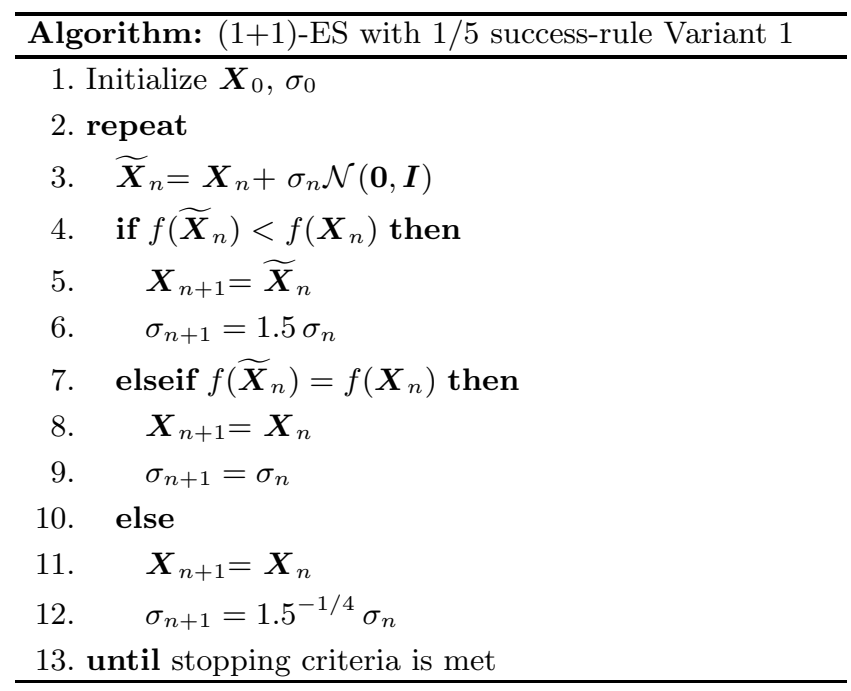

Table 3: Pseudo-code for the $(1+1)$-ES with onefifth success rule Variant 2.

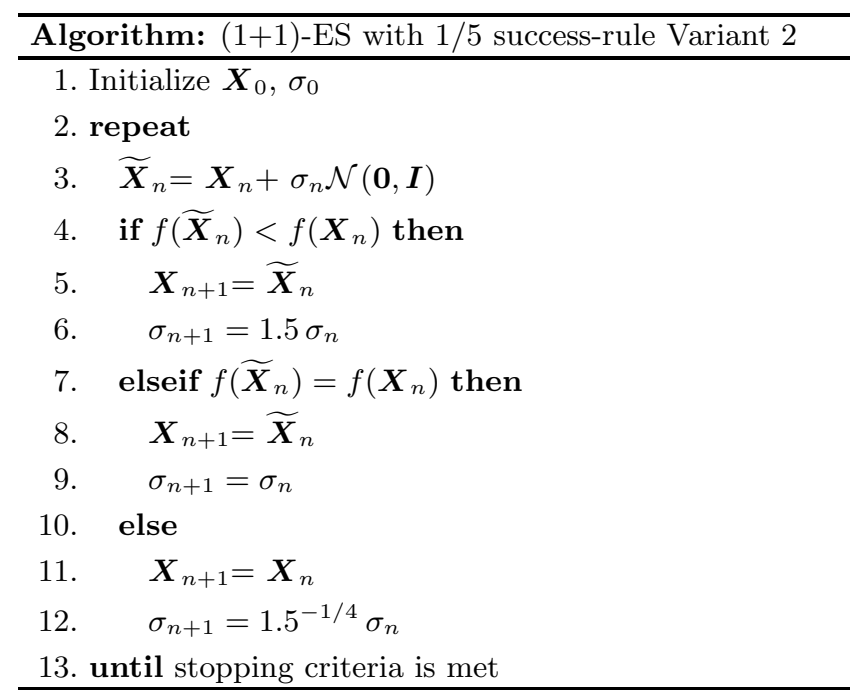

This process is iterated. Whenever the overall number of function evaluations reaches $10^{6} \mathrm{D}$ or an objective function value below the target function value is reached, the algorithm is stopped.

\subsection{Stopping criteria for single runs}

A single run of the $(1+1)$-ES is terminated when one of the following condition is satisfied:

- TolSigma $=10^{-15}$ : stop if $\sigma_{n} \leq$ TolSigma

- MaxNoImp $=4 * 4 \frac{\ln (10)}{\ln (1.5)}$ : stop if there was no improvements during MaxNoImp successive iterations (i.e.lower value found)of the objective function 


\section{PARAMETER TUNING}

No specific parameter tuning has been done, several trials on the whole testbed have been done to determine the value for TolSigma since some previous values set by the author turned out to be too large (typically $10^{-8}$ is too large) and did not allow to observe convergence on the Attractive Sector function. The same settings have been used for all functions such that the crafting effort [5] computes to $\operatorname{Cr} E=0$.

\section{CPU TIMING EXPERIMENT}

For the timing experiment the $(1+1)-\mathrm{ES}$ with independent restarts was run with a maximum of $10^{5} \mathrm{D}$ function evaluations and restarted until 30 seconds has passed (according to Figure 2 in [5]). The experiments have been conducted with an Intel Pentium $4 \mathrm{CPU} 3.80 \mathrm{GHz}$ under Linux using a C-implementation. The time per function evaluation was $7.5 ; 9.9 ; 15 ; 27 ; 51 ; 97$ times $10^{-7}$ seconds in dimensions 2 ; $3 ; 5 ; 10 ; 20 ; 40$ respectively.

\section{RESULTS}

Results from experiments according to [5] on the benchmark functions given in $[4,6]$ are presented in Figures 1 and 2 and in Table 4.

Among the 24 functions, 13 were solved in 5-D and 9 in 20 D. Not too surprisingly, the $(1+1)$-ES can solve all moderate functions in 5-D and 3 out of 4 in 20-D with an ERT of less than $10^{6} \mathrm{D}$. For the ill-conditioned problems, the picture is different since only 2 out of 5 problems are solved in 20-D and 1 in 5 -D. Furthermore solving ill-conditioned problems requires an ERT larger than $6 \times 10^{5} \mathrm{D}$ though most of the moderate problems can be solved with an ERT smaller than $10^{5} \mathrm{D}$. Besides, though theoretically, the $(1+1)-\mathrm{ES}$ should also solve the Ellipsoid function if the maximum number of evaluations would be large enough, we have observed that due to numerical precisions, the $(1+1)$-ES cannot solve it even after increasing the maximum number of evaluations. The bad results on ill-conditioned problems are of course not surprising because the method has no mechanism to deform the sampling distribution, as opposed to the famous Covariance Matrix Algorithm [7].

Furthermore, we can observe a consequence of the invariance of the algorithm with respect to the coordinate system: the performances are invariant on the original / rotated Ellipsoid, Rosenbrock, Rastrigin functions.

The performance is poor on multi-modal functions, however the Gallagher multimodal functions are solved.

The Variant 1 and 2 gave slightly worse results. In particular on the step-ellipsoid where increasing the step-size is beneficial for facing the plateaus.

\section{DISCUSSION}

The simple (1+1)-ES with one-fifth success rule with a restart mechanism is able to solve 13 (resp. 9) functions in 5-D (resp. 20-D) but performs poorly on ill-conditioned problems. This is to be expected from the lack of adaptation mechanism for the covariance matrix and can be improved by introducing such a mechanism [1]. The performance is poor on multi-modal functions. The results are foreseen to generalize well due to two invariance properties of the algorithm: invariance to order-preserving transformations of the function value and rotational invariance.

\section{Acknowledgments}

The author would like to acknowledge Nikolaus Hansen, Steffen Finck, Raymond Ros for their great and hard work on the BBOB project, Nikolaus Hansen and Marc Schoenauer for their kind and persistent support and Dimo Brockhoff for his comments on the paper.

\section{REFERENCES}

[1] A. Auger and N. Hansen. Benchmarking the $(1+1)$-CMA-ES on the BBOB-2009 Function Testbed. In Workshop Proceedings of the Genetic and Evolutionary Computation Conference (GECCO 2009). ACM Press, 2009.

[2] S. H. Brooks. A discussion of random methods for seeking maxima. Operations Research, 6:244- 251, 1958.

[3] L. Devroye. The compund random search. In International Symposium on Systems Engineering and Analysis, pages 195-110. Purdue University, 1972.

[4] S. Finck, N. Hansen, R. Ros, and A. Auger. Real-parameter black-box optimization benchmarking 2009: Presentation of the noiseless functions. Technical Report 2009/20, Research Center PPE, 2009.

[5] N. Hansen, A. Auger, S. Finck, and R. Ros. Real-parameter black-box optimization benchmarking 2009: Experimental setup. Technical Report RR-6828, INRIA, 2009.

[6] N. Hansen, S. Finck, R. Ros, and A. Auger. Real-parameter black-box optimization benchmarking 2009: Noiseless functions definitions. Technical Report RR-6829, INRIA, 2009.

[7] N. Hansen and A. Ostermeier. Completely derandomized self-adaptation in evolution strategies. Evolutionary Computation, 9(2):159-195, 2001.

[8] S. Kern, S. Müller, N. Hansen, D. Büche, J. Ocenasek, and P. Koumoutsakos. Learning Probability Distributions in Continuous Evolutionary Algorithms A Comparative Review. Natural Computing, 3(1):77-112, 2004.

[9] I. Rechenberg. Evolutionsstrategie. Friedrich Frommann Verlag (Günther Holzboog KG), Stuttgart, 1973.

[10] M. Schumer and K. Steiglitz. Adaptive step size random search. Automatic Control, IEEE Transactions on, 13:270-276, 1968. 

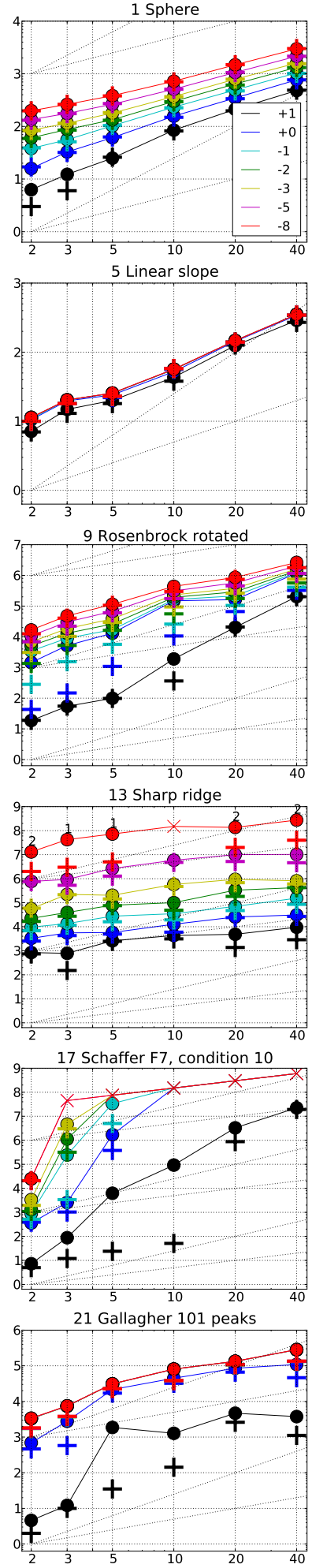
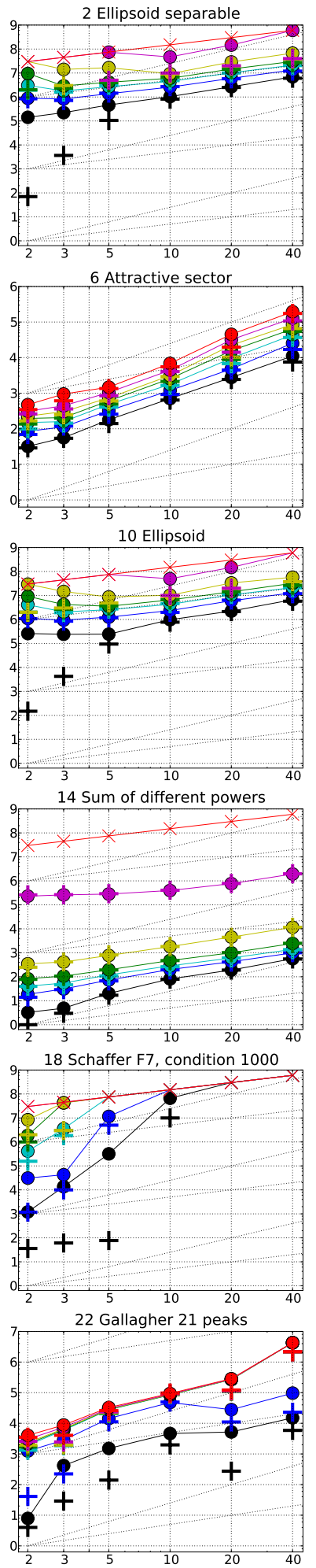
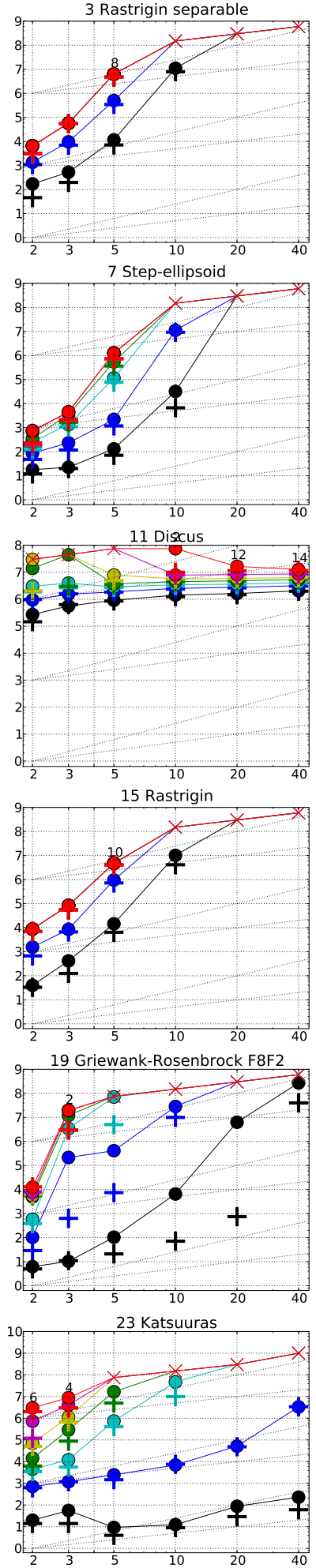
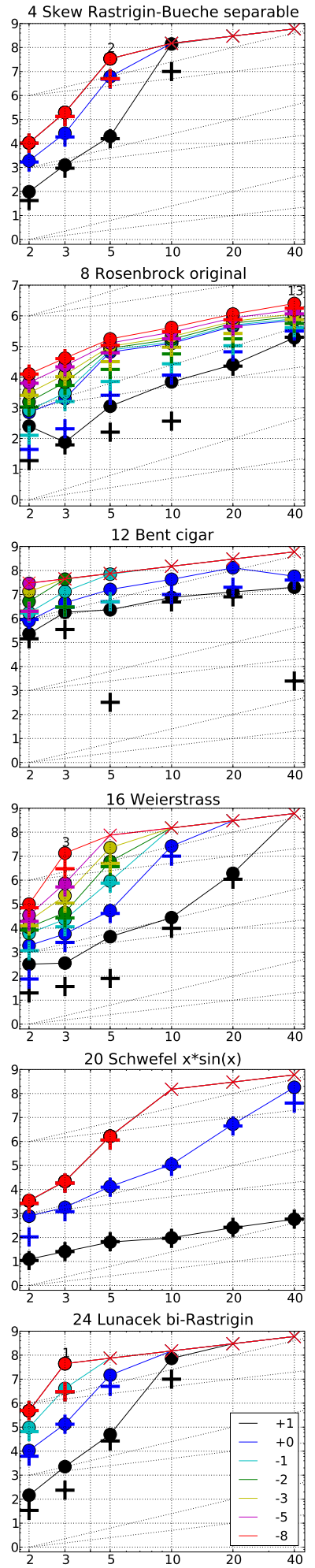

Figure 1: Expected Running Time (ERT, ๑) to reach $f_{\mathrm{opt}}+\Delta f$ and median number of function evaluations of successful trials $(+)$, shown for $\Delta f=10,1,10^{-1}, 10^{-2}, 10^{-3}, 10^{-5}, 10^{-8}$ (the exponent is given in the legend of $f_{1}$ and $\left.f_{24}\right)$ versus dimension in log-log presentation. The $\operatorname{ERT}(\Delta f)$ equals to \#FEs $(\Delta f)$ divided by the number of successful trials, where a trial is successful if $f_{\mathrm{opt}}+\Delta f$ was surpassed during the trial. The \#FEs $(\Delta f)$ are the total number of function evaluations while $f_{\text {opt }}+\Delta f$ was not surpassed during the trial from all respective trials (successful and unsuccessful), and $f_{\text {opt }}$ denotes the optimal function value. Crosses $(\times)$ indicate the total number of function evaluations \#FEs $(-\infty)$. Numbers above ERT-symbols indicate the number of successful trials. Annotated numbers on the ordinate are decimal logarithms. Additional grid lines show linear and quadratic scaling. 

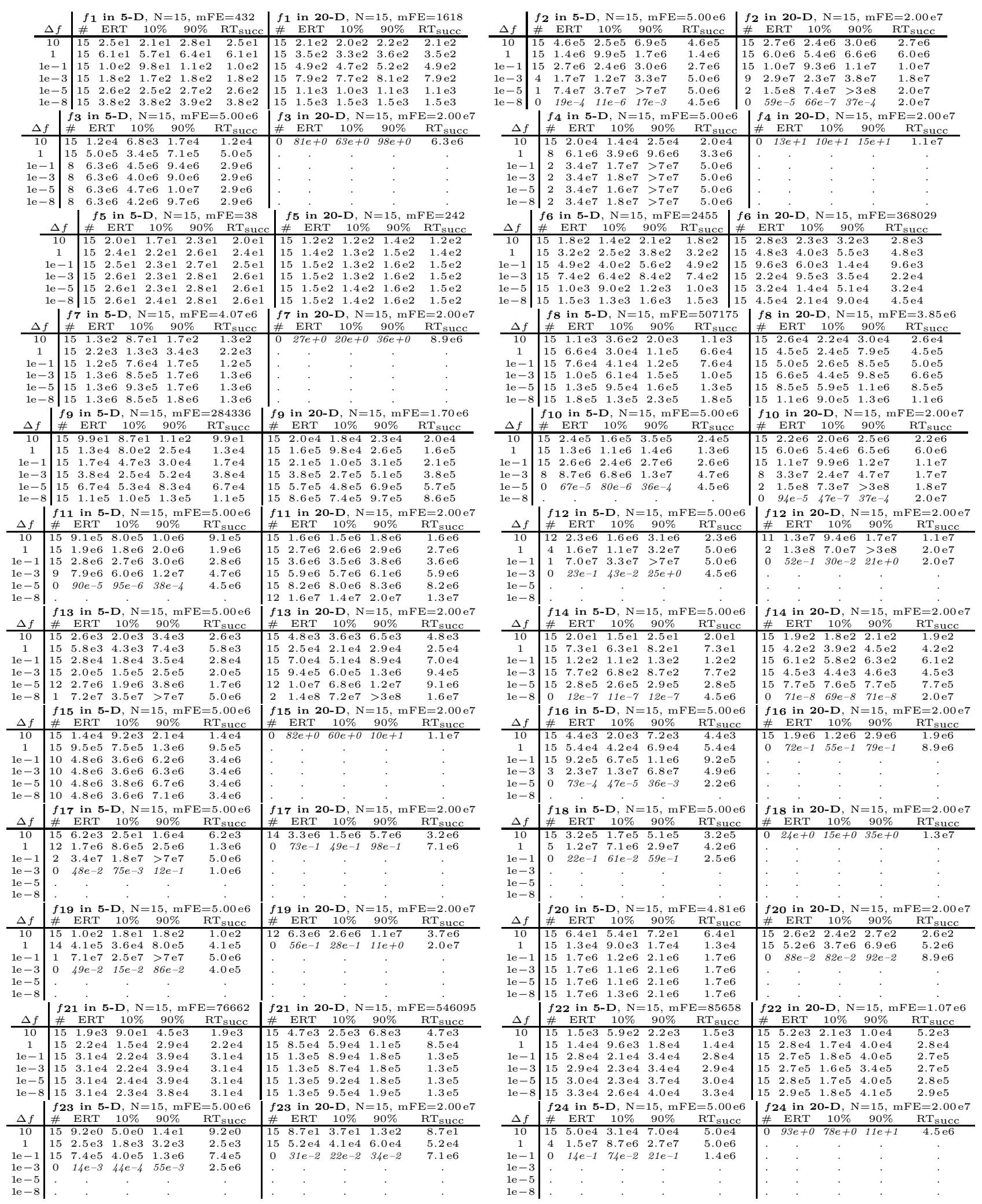

Table 4: Shown are, for a given target difference to the optimal function value $\Delta f:$ the number of successful trials $(\#)$; the expected running time to surpass $f_{\text {opt }}+\Delta f$ (ERT, see Figure 1 ); the 10\%-tile and 90\%-tile of the bootstrap distribution of ERT; the average number of function evaluations in successful trials or, if none was successful, as last entry the median number of function evaluations to reach the best function value $\left(\mathrm{RT}_{\text {succ }}\right)$. If $f_{\mathrm{opt}}+\Delta f$ was never reached, figures in italics denote the best achieved $\Delta f$-value of the median trial and the $10 \%$ and $90 \%$-tile trial. Furthermore, $\mathrm{N}$ denotes the number of trials, and $\mathrm{mFE}$ denotes the maximum of number of function evaluations executed in one trial. See Figure 1 for the names of functions. 

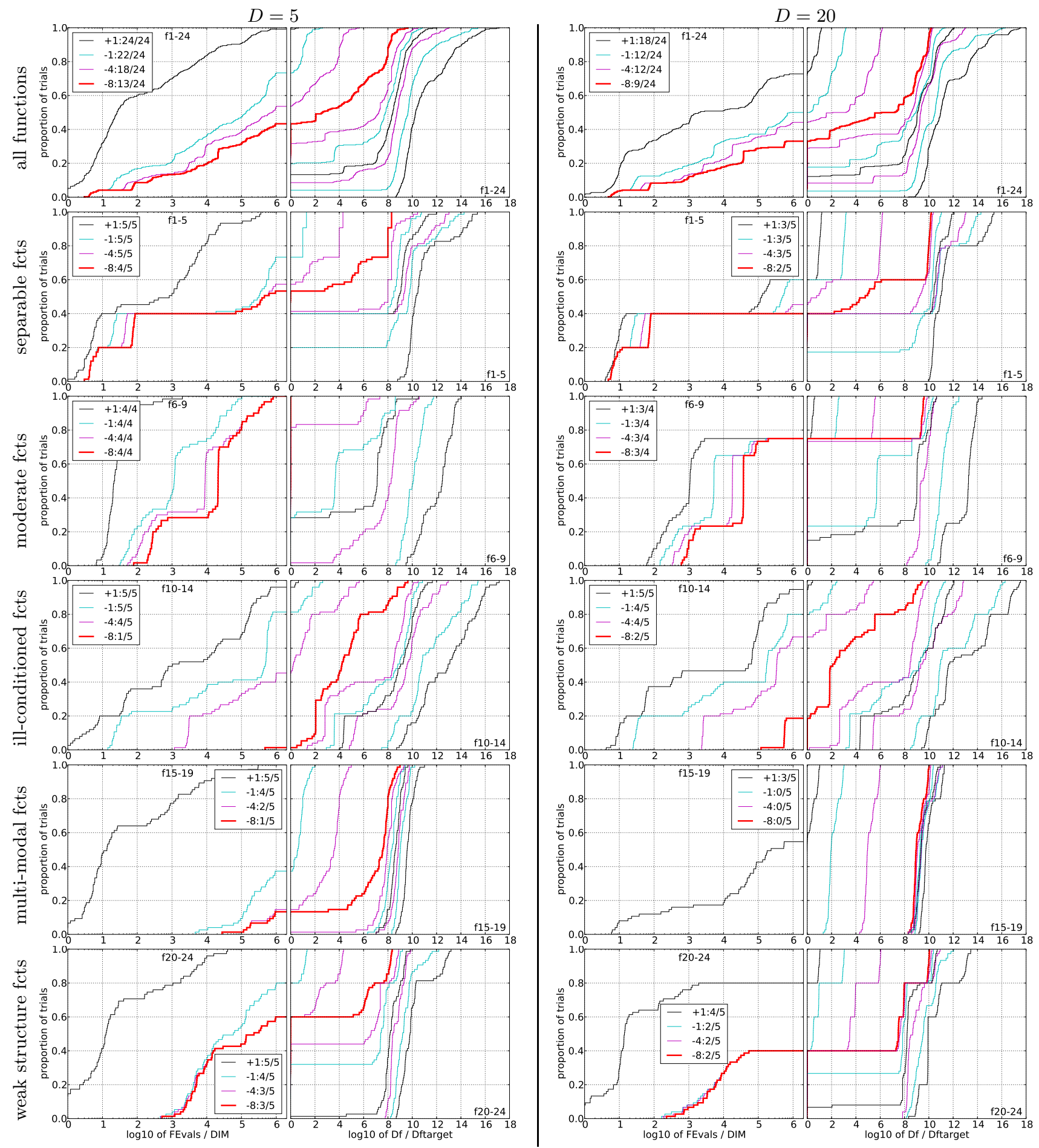

Figure 2: Empirical cumulative distribution functions (ECDFs), plotting the fraction of trials versus running time (left subplots) or versus $\Delta f$ (right subplots). The thick red line represents the best achieved results. Left subplots: ECDF of the running time (number of function evaluations), divided by search space dimension $D$, to fall below $f_{\text {opt }}+\Delta f$ with $\Delta f=10^{k}$, where $k$ is the first value in the legend. Right subplots: ECDF of the best achieved $\Delta f$ divided by $10^{k}$ (upper left lines in continuation of the left subplot), and best achieved $\Delta f$ divided by $10^{-8}$ for running times of $D, 10 \mathrm{D}, 100 \mathrm{D} \ldots$ function evaluations (from right to left cycling blackcyan-magenta). Top row: all results from all functions; second row: separable functions; third row: misc. moderate functions; fourth row: ill-conditioned functions; fifth row: multi-modal functions with adequate structure; last row: multi-modal functions with weak structure. The legends indicate the number of functions that were solved in at least one trial. FEvals denotes number of function evaluations, $D$ and DIM denote search space dimension, and $\Delta f$ and Df denote the difference to the optimal function value. 